\title{
Prevalence and Predictors of Current Tobacco Smoking in Bangladesh
}

\author{
Sultana $\mathrm{P}^{* 1}$, Akter $\mathrm{S}^{2}$, Rahman $\mathrm{MM}^{3}$ and Alam MS ${ }^{1}$ \\ ${ }^{1}$ Department of Statistics, University of Rajshahi, Bangladesh \\ ${ }^{2}$ Department of Epidemiology and Prevention, Clinical Research Center, National Center for Global Health and \\ Medicine, Tokyo, Japan \\ ${ }^{3}$ Department of Global Health Policy, The University of Tokyo, Japan, and Department of Population Science and \\ Human Resource Development, University of Rajshahi, Bangladesh
}

${ }^{*}$ Corresponding author: Sultana P, Department of Statistics, University of Rajshahi, Bangladesh, Tel: +88 01914254247, E-mail: papia.stat@ymail.com

\section{Citation: Sultana P, Akter S, Rahman MM, Alam MS (2015) Prevalence and Predictors of Current Tobacco Smoking in Bangladesh. J Biostat Biometric App 1(1): 102. doi: 10.15744/2455-765X.1.102}

Received Date: February 16, 2015 Accepted Date: May 19, 2015 Published Date: May 21, 2015

\begin{abstract}
Introduction: Tobacco is identified as leading modifiable global disease risk factor. The objective of this paper is to estimate the prevalence, and to identify the socioeconomic and demographic correlates of tobacco smoking in Bangladesh.

Data and Methodology: We have used secondary data of size 9629 (male=4468 and female=5161) aged 15 years and above collected by the Global Adult Tobacco Survey (GATS), 2010. Principle Component Analysis (PCA) has been used to get the wealth index. Binary logistic regression model has been used to assess the predictors of current tobacco smoking.

Results: Prevalence of current tobacco smokers in Bangladesh was 23.19 (48.28\% male and 1.47\% female) and the prevalence of current daily tobacco smokers was 21.16. Rural respondents were significantly more likely to smoke tobacco currently. Comparative to females, males were more likely to smoke currently (adjusted $\mathrm{OR}=37.55,95 \% \mathrm{CI}=25.91,54.41$ ). Respondents of youngest age group (15-24 yrs) were less likely to smoke currently than all other age groups and respondents with no formal schooling were more likely to smoke than respondents with all other levels of education. Businessmen, farmers and workers/day labours were more likely to smoke, and employee, students and respondents with other jobs were less likely to smoke. It was also found that respondents with lowest wealth index were most likely to smoke and respondents with higher wealth index were least likely to smoke.

Conclusion: The results reveal that in Bangladesh, tobacco smoking is strongly associated with social disadvantage, for example, low socio-economic status, less education, stressed or low-paid job, etc. Giving it as public health priority, WHO Framework Convention on Tobacco Control should be implemented. In addition, a nationwide campaign is needed to educate people in rural area about the health risks of smoking tobacco.
\end{abstract}

Keywords: Prevalence; Predictor; Odds Ratio; Logistic Regression; Tobacco Smoking; GATS

\section{Introduction}

Tobacco smoking is a leading modifiable global disease risk factor, with nearly 6 million premature deaths, $6.90 \%$ of years of life lost, and 5.5\% disability-adjusted life-years (DALYs) in 2010 [1,2]. Global age-standardized prevalence of daily tobacco smoking was $31.1 \%$ in 2012 for men [3]. Nearly $80 \%$ of the more than one billion smokers worldwide live in low- and middle-income countries, where the burden of tobacco-related illness and death is heaviest [4]. Given the importance of tobacco as a risk to health, monitoring the distribution and intensity of tobacco use is critical particularly for low- and middle- income countries.

Bangladesh is a low-income country and one of the largest tobacco consuming countries in the world [3]. According to a previous study of Bangladesh, smoking causes about $25 \%$ of all deaths in Bangladeshi men aged 25 to 69 years and an average loss of seven years of life per smoker [5]. Tobacco-use results in both health and economic costs that is large and growing [6,7]. Due to its easy accessibility and social acceptability, there are now more young women and teenagers having access to cigarettes and hence getting addicted. Its losses are immeasurable or uncountable. Some losses are directly related and others are related indirectly. Smoking affects individual smoker, his/her family and society as a whole. Significant costs are being used for medicine purpose. It reduces the strength of individuals working capacity. As tobacco smoking is becoming a threat of the health of population and an economic burden, use of tobacco is not stopped and no effective anti-smoking efforts are made in Bangladesh.

To date, some studies have been conducted in Bangladesh to investigate the prevalence and predictors of tobacco smoking [811]. However, these previous studies has been limited to small sample size [8], specific gender [8-11], urban smokers [8,9], or adolescent population [9]. The aims of this study, therefore, were to obtain a nationally representative estimate of prevalence of smoking in Bangladesh, and to identify socio-economic and demographic correlates of current tobacco smokers. 


\section{Data and Methodology}

We have used secondary data collected by the Global Adult Tobacco Survey (GATS), 2010 [12]. The survey was conducted in 14 countries including Bangladesh, Brazil, China, Egypt, India, Mexico, Philippines, Poland, Russia, Thailand, Turkey, Ukraine, Uruguay and Vietnam from 2008 to 2010. GATS used a global standardized methodology. It included information on the respondents' background characteristics, tobacco use (smoking and smokeless), cessation, second-hand smoke, economics, media, and knowledge, attitudes and perceptions of tobacco use. In Bangladesh, GATS was conducted in 2009 as a household survey of persons 15 years of age or older by the National Institute of Preventive and Social Medicine in collaboration with the Bangladesh Bureau of Statistics (BBS) and National Institute of Population Research and Training (NIPRT). A multi-stage (three-stage), geographically clustered sample design was used to produce nationally representative data. At the first stage 400 Primary Sampling Units (PSU)s (Mauza in rural and Mohalla in urban areas) were selected with probability proportional to size (PPS), followed by a random selection of one Secondary Sampling Unit (SSU) per selected PSU. At the third stage households were selected systematically within the listed households from a selected SSU. One individual was randomly chosen from each selected household to participate in the survey. Survey information was collected using handheld devices. The household response rate was $97.7 \%$, the individual response rate was $95.8 \%$ and the overall response rate was $93.6 \%$. There were a total of 9629 completed interviews (male $=4468$ and female =5161). Details about the survey methods, questionnaire, and definitions of various terminologies can be found in [12-14].

The wealth index was constructed by the GATS Collaborator Team using principal component analysis. Asset information covered household ownership of a number of items, such as electricity, flush toilet, fixed telephone, cell telephone, television, radio, refrigerator, car, moped/scooter/motorcycle, washing machine, bicycle, sewing machine, almirah/ wardrobe, table, bed or cot, chair or bench, watch or clock, as well as the type of main material used for the roof of the main house (cement, tin and katcha such as bamboo/thatched/straw). Each asset was assigned a weight (factor score) generated through principal components analysis, and the resulting asset scores were standardized in relation to a normal distribution with a mean of zero and standard deviation of one. Each household was then assigned a score for each asset, and the scores were summed for each household; individuals were ranked according to the total score of the household in which they resided. The sample was then divided into quintiles from one (lowest) to five (highest). A single asset index was developed for the whole sample; indices were not prepared for urban and rural populations separately [15].

The term 'current tobacco smokers' includes 'daily' and/ or 'occasional (less than daily)' tobacco smokers. Ex-smokers have not been included in the analysis of this paper.

\section{Statistical Methods}

Various statistical methodologies have been used to analyze the data. Descriptive analysis has been performed to know the characteristics of the study subjects. For that frequency with percentage has been reported. A comparison of socio-demographic and economic characteristics of study subjects to current tobacco smokers has been performed. To compare variables chi-square test (Pearson Chi-square or Likelihood Ratio Chi-square) has been used as they are categorical, and prevalence with $95 \%$ confidence interval has been reported for individual variable. These tests have been performed at $5 \%$ level of significance. To analyze the sociodemographic and economic predictors to current tobacco smoker's binary logistic regression has been used and unadjusted and adjusted Odds Ratio (OR) has been reported. Age adjusted and unadjusted prevalence has been reported, too. Statistical software StataSE version 11 (StataCorp, USA) has been used to carry out statistical analyses. Missing data on "age" and "occupation" has been adjusted using related information. For example, missing data on age has been adjusted using year of birth; missing data on occupation has been adjusted using information on educational status and age, those were for students and elderly persons, only. However, missing data on educational level (0.66\%) cannot be adjusted as no other supportive variable is available. For advanced analysis missing data has been avoided and thus sample size 9565 has been used in the advanced analysis.

\section{Results}

It has been observed that prevalence of current tobacco smokers in Bangladesh is 23.19 (48.28\% male and $1.47 \%$ female) and the prevalence of current daily tobacco smokers is 21.16 (Table 1). Table 2 presents percentage of various tobacco products smoked in Bangladesh. It has been found that among the tobacco products manufactured cigarettes and bidis are mostly smoked. Equivalent numbers of subjects were surveyed from rural and urban area (50.44\% from urban and 40.56\% from rural) (Table 3 ). Among the survey subjects, females are more than males (46.40\% male and 53.60\% female). Most of the subjects are of no formal schooling (35.48\%) and most are of homemaker/ housework (41.90\%).

Most Prevalence of tobacco smoking is relatively higher in rural area $(52.35 \%$; $95 \% \mathrm{CI}=50.29,54.42)$ than urban area $(47.65 \%$; $95 \%$ $\mathrm{CI}=45.58,49.72$ ) (Table 4). Maximum of the tobacco smokers belong to age group 25-34 years $(26.29 \%$; $95 \% \mathrm{CI}=24.46,28.11)$ and $35-44$ years $(28.93 \%$; 95\% CI=27.05, 30.81). Respondents with business (small/large) as main occupation smoke most $(21.41 \%$, $95 \% \mathrm{CI}=19.70,23.11)$. On the other hand, respondents who are homemaker/housework smoke least $(2.28 \%, 95 \% \mathrm{CI}=1.66,2.90)$. It has been also found that respondents who belongs to highest wealth index, smoke tobacco least. However, educational level, independently is not gradually decreasing the smoking of tobacco which was expected, although it differs significantly to smoking status ( $\mathrm{p}$-value $<0.001$ ). 


\begin{tabular}{|c|c|c|c|c|}
\hline \multirow{4}{*}{ Daily } & & Male (\%) & Female (\%) & Total (\%) \\
\hline & Urban & 41.24 & 0.86 & 19.85 \\
\cline { 2 - 5 } & Rural & 47.16 & 1.70 & 22.51 \\
\cline { 2 - 5 } & p-value & $<0.001$ & 0.007 & 0.001 \\
\cline { 2 - 5 } & Total & 44.14 & 1.28 & 21.16 \\
\hline \multirow{5}{*}{ Less than daily } & Urban & 4.20 & 0.16 & 2.06 \\
\cline { 2 - 5 } & Rural & 4.08 & 0.23 & 1.99 \\
\cline { 2 - 5 } & p-value & 0.830 & 0.533 & 0.812 \\
\cline { 2 - 5 } & Total & 4.14 & 0.19 & 2.03 \\
\hline \multirow{5}{*}{ Current smokers } & Urban & 45.45 & 1.01 & 21.91 \\
\cline { 2 - 5 } & Rural & 51.24 & 1.93 & 24.50 \\
\cline { 2 - 5 } & p-value & 0.006 & $<0.001$ & 0.003 \\
\cline { 2 - 5 } & Total & 48.28 & 1.47 & 23.19 \\
\hline \multirow{7}{*}{ Not at all } & Urban & 54.55 & 98.99 & 78.09 \\
\cline { 2 - 5 } & Rural & 48.76 & 98.07 & 75.50 \\
\cline { 2 - 5 } & Total & 51.72 & 98.85 & 76.81 \\
\hline
\end{tabular}

Note: Total sample size $=9629$. P-values are for the difference of proportions for residence

Table 1: Prevalence of current tobacco smoking in Bangladesh, 2010 stratified by area of residence and gender

\begin{tabular}{|l|c|c|c|}
\hline Type of Tobacco Product smoked & Rural & Urban & Total \\
\hline Manufactured cigarette & 10.98 & 16.70 & 13.86 \\
\hline Bidi & 13.89 & 4.98 & 9.40 \\
\hline Water pipe (Hukkah) & 0.73 & 1.03 & 0.88 \\
\hline Hand-rolled cigarette & 0.23 & 0.84 & 0.54 \\
\hline Pipes full of tobacco & 0.25 & 0.78 & 0.52 \\
\hline Cigars, cheroots, or cigarillos & 0.19 & 0.84 & 0.52 \\
\hline Other smoked tobacco & 0.52 & 0.80 & 0.66 \\
\hline
\end{tabular}

Note: Total sample size $=9629$. Other smoked tobacco includes zarda, pan masala, etc

Table 2: Prevalence of tobacco product smoked in Bangladesh, 2010 stratified by area of residence

\begin{tabular}{|l|l|}
\hline Socio-demographic characteristics & n (\%) Sample size=9629 \\
\hline Residence & \\
\hline Urban & $4857(50.44)$ \\
\hline Rural & $4772(49.56)$ \\
\hline Gender & \\
\hline Male & $4468(46.40)$ \\
\hline Female & $5161(53.60)$ \\
\hline Age (years) & \\
\hline $15-24$ & $2073(21.53)$ \\
\hline $25-34$ & $2665(27.68)$ \\
\hline $35-44$ & $2232(23.18)$ \\
\hline $45-54$ & $1329(13.80)$ \\
\hline $55 \&$ above & $1330(13.81)$ \\
\hline Educational Level & \\
\hline No formal schooling & $3416(35.48)$ \\
\hline Less than primary school completed & $1487(15.44)$ \\
\hline Primary school completed & $1115(11.58)$ \\
\hline Less than secondary school completed & $1937(20.12)$ \\
\hline Secondary school completed & $663(6.89)$ \\
\hline High school completed & $463(4.81)$ \\
\hline College/University completed & $273(2.84)$ \\
\hline Post graduate degree completed & $211(2.19)$ \\
\hline Missing & $64(0.66)$ \\
\hline
\end{tabular}




\begin{tabular}{|l|l|}
\hline Socio-demographic characteristics & n (\%) Sample size=9629 \\
\hline Occupation & \\
\hline Government employee & $221(2.30)$ \\
\hline Non-Government employee & $740(7.69)$ \\
\hline Business-small & $865(8.98)$ \\
\hline Business-large & $128(1.33)$ \\
\hline Farming (land owner \& farmer) & $826(8.58)$ \\
\hline Agricultural worker & $374(3.88)$ \\
\hline Industrial worker & $214(2.22)$ \\
\hline Daily laborer & $631(6.55)$ \\
\hline Other self-employed & $318(3.30)$ \\
\hline Student & $463(4.81)$ \\
\hline Homemaker/Housework & $4030(41.85)$ \\
\hline Retired & $113(1.17)$ \\
\hline Unemployed, able to work & $153(1.59)$ \\
\hline Unemployed, unable to work & $165(1.71)$ \\
\hline Other (specify) & $388(4.03)$ \\
\hline Wealth index & $1866(19.38)$ \\
\hline Lowest & $2068(21.48)$ \\
\hline Low & $1732(17.99)$ \\
\hline Middle & $2040(21.19)$ \\
\hline High & $1923(19.97)$ \\
\hline Highest & \\
\hline & \\
\hline
\end{tabular}

Note: Wealth index was calculated using principal component analysis. Asset information covered household ownership of a number of items, such as electricity, flush toilet, fixed telephone, cell telephone, television, radio, refrigerator, car, moped/scooter/motorcycle, washing machine, bicycle, sewing machine, almirah/ wardrobe, table, bed or cot, chair or bench, watch or clock, as well as the type of main material used for the roof of the main house (cement, tin and katcha such as bamboo/thatched/straw)

Table 3: Socio-demographic and economic characteristics of the study subjects

\begin{tabular}{|c|c|c|c|}
\hline \multirow[t]{3}{*}{ Socio-demographic factors } & \multicolumn{2}{|c|}{ Current Smoking Status } & \multirow[b]{3}{*}{ P-value ${ }^{\star}$} \\
\hline & Smokers & Non-Smokers & \\
\hline & Prevalence (95\% CI) & Prevalence $(95 \%$ CI $)$ & \\
\hline \multicolumn{4}{|l|}{ Residence } \\
\hline Urban & $47.65(45.58,49.72)$ & $51.28(50.15,52.42)$ & 0.003 \\
\hline \multicolumn{4}{|l|}{ Gender } \\
\hline Male & $96.60(95.84,97.35)$ & $31.25(30.19,32.30)$ & $<0.001$ \\
\hline \multicolumn{4}{|l|}{ Age (years) } \\
\hline $15-24$ & $10.39(9.12,11.66)$ & $24.89(23.91,25.88)$ & \multirow{5}{*}{$<0.001$} \\
\hline $25-34$ & $26.29(24.46,28.11)$ & $28.10(27.07,29.12)$ & \\
\hline $35-44$ & $28.93(27.05,30.81)$ & $21.44(20.51,22.38)$ & \\
\hline $45-54$ & $18.14(16.54,19.74)$ & $12.49(11.74,13.25)$ & \\
\hline $55 \&$ above & $16.26(14.73,17.79)$ & $13.07(12.31,13.84)$ & \\
\hline \multicolumn{4}{|l|}{ Educational Level } \\
\hline No formal schooling & $46.08(44.01,48.15)$ & $32.27(31.21,33.34)$ & \multirow{8}{*}{$<0.001$} \\
\hline Less than primary school completed & $17.91(16.32,19.50)$ & $14.70(13.89,15.50)$ & \\
\hline Primary school completed & $8.91(7.73,10.09)$ & $12.39(11.63,13.14)$ & \\
\hline Less than secondary school completed & $15.81(14.29,17.32)$ & $21.42(20.48,22.35)$ & \\
\hline Secondary school completed & $4.52(3.66,5.39)$ & $7.60(6.99,8.20)$ & \\
\hline High school completed & $2.78(2.09,3.46)$ & $5.42(4.91,5.94)$ & \\
\hline College/University completed \& higher & $4.00(3.17,4.80)$ & $5.34(4.83,5.85)$ & \\
\hline Missing & -- & $0.87(0.65,1.08)$ & \\
\hline
\end{tabular}




\begin{tabular}{|c|c|c|c|}
\hline \multirow[t]{3}{*}{ Socio-demographic factors } & \multicolumn{2}{|c|}{ Current Smoking Status } & \multirow[b]{3}{*}{ P-value ${ }^{*}$} \\
\hline & Smokers & Non-Smokers & \\
\hline & Prevalence $(95 \% \mathrm{CI})$ & Prevalence $(95 \% \mathrm{CI})$ & \\
\hline \multicolumn{4}{|l|}{ Occupation (\%) } \\
\hline Employment (government, non-government) & $11.69(10.36,13.02)$ & $9.46(8.80,10.13)$ & \multirow{7}{*}{$<0.001$} \\
\hline Business (small/large) & $21.41(19.70,23.11)$ & $6.96(6.38,7.54)$ & \\
\hline Farming (land owner \& farmer) & $19.66(18.01,21.31)$ & $5.23(4.72,5.74)$ & \\
\hline $\begin{array}{l}\text { Agrior industrial worker/Daily labour/Other self- } \\
\text { employed }\end{array}$ & $34.08(32.11,36.05)$ & $10.49(9.79,11.19)$ & \\
\hline Homemaker/ Housework & $2.28(1.66,2.90)$ & $53.80(52.66,54.94)$ & \\
\hline Retired and unemployed (able/unable to work) & $4.48(3.62,5.34)$ & $4.48(4.00,4.95)$ & \\
\hline Student/ Others & $6.40(5.39,7.42)$ & $9.57(8.90,10.24)$ & \\
\hline \multicolumn{4}{|l|}{ Wealth index } \\
\hline Lowest & $23.51(21.75,25.27)$ & $18.13(17.25,19.01)$ & \multirow{5}{*}{$<0.001$} \\
\hline Low & $25.44(23.63,27.24)$ & $20.28(19.36,21.19)$ & \\
\hline Middle & $18.00(16.41,19.60)$ & $17.98(17.11,18.86)$ & \\
\hline High & $19.48(17.84,21.12)$ & $21.70(20.76,22.64)$ & \\
\hline Highest & $13.57(12.15,14.99)$ & $21.90(20.96,22.85)$ & \\
\hline
\end{tabular}

${ }^{*}$ P-values are obtained from Chi-square test (Pearson Chi-square or Likelihood Ratio whichever applicable), $95 \%$ CIs are obtained from $\mathrm{p} \pm 1.96$ se(p). - indicates N/A due to no observation. Total sample size $=9629$.

Table 4: Comparing socio-demographic and economic factors between smokers and non-smokers

Age adjusted and unadjusted prevalence of current tobacco smokers with 95\% CI have been reported, too (Table 5). It has been found that both age-adjusted and unadjusted prevalence of current smoking are similar for all cases. About $48.28 \%$ of males are currently smoking and about $1.49 \%$ of females are currently smoking. About $22.02 \%$ of urban people are currently smoking, whereas about $24.70 \%$ of rural people are currently smoking.

\begin{tabular}{|c|c|c|}
\hline \multirow[t]{2}{*}{ Socio-demographic factors } & \multicolumn{2}{|c|}{ Current Smokers } \\
\hline & $\begin{array}{c}\text { Prevalence }(95 \% \mathrm{CI}) \\
\text { Unadjusted }\end{array}$ & $\begin{array}{c}\text { Prevalence }(95 \% \mathrm{CI}) \\
\text { Adjusted }\end{array}$ \\
\hline \multicolumn{3}{|l|}{ Residence } \\
\hline Urban & $22.02(20.85,23.19)$ & $22.34(21.18,23.52)$ \\
\hline Rural & $24.70(23.47,25.93)$ & $24.34(23.13,25.55)$ \\
\hline \multicolumn{3}{|l|}{ Gender } \\
\hline Female & $1.49(1.16,1.82)$ & $1.52(1.18,1.86)$ \\
\hline Male & $48.28(46.81,49.74)$ & $47.67(46.21,49.14)$ \\
\hline \multicolumn{3}{|l|}{ Educational Level } \\
\hline No formal schooling & $30.12(28.58,31.66)$ & $28.34(26.80,29.88)$ \\
\hline Less than primary school completed & $26.90(24.65,29.15)$ & $27.09(24.83,29.34)$ \\
\hline Primary school completed & $17.85(15.60,20.10)$ & $18.61(16.29,20.93)$ \\
\hline Less than secondary school completed & $18.22(16.50,19.94)$ & $19.52(17.68,21.35)$ \\
\hline Secondary school completed & $15.23(12.50,17.97)$ & $16.30(13.41,19.19)$ \\
\hline High school completed & $13.39(10.29,16.49)$ & $14.14(10.90,17.38)$ \\
\hline College/University completed\& higher & $18.39(14.94,21.84)$ & $18.18(14.78,21.59)$ \\
\hline \multicolumn{3}{|l|}{ Occupation } \\
\hline Employment (government, non-government) & $27.30(24.48,30.13)$ & $28.00(25.16,30.84)$ \\
\hline Business (small/large) & $48.14(45.03,51.24)$ & $47.40(44.33,50.47)$ \\
\hline Farming (land owner \& farmer) & $53.15(49.74,56.55)$ & $50.06(46.60,53.51)$ \\
\hline $\begin{array}{l}\text { Agri or industrial worker/Daily labour/Other } \\
\text { self-employed }\end{array}$ & $49.90(47.39,52.41)$ & $50.32(47.84,52.80)$ \\
\hline Homemaker/ Housework & $1.28(0.93,1.63)$ & $1.31(0.95,1.67)$ \\
\hline Retired and unemployed (able/unable to work) & $23.53(19.50,27.56)$ & $17.55(14.07,21.04)$ \\
\hline Student/ Others & $16.74(14.24,19.25)$ & $19.45(16.58,22.31)$ \\
\hline
\end{tabular}




\begin{tabular}{|c|c|c|}
\hline \multirow[t]{2}{*}{ Socio-demographic factors } & \multicolumn{2}{|c|}{ Current Smokers } \\
\hline & $\begin{array}{c}\text { Prevalence }(95 \% \mathrm{CI}) \\
\text { Unadjusted }\end{array}$ & $\begin{array}{c}\text { Prevalence }(95 \% \text { CI }) \\
\text { Adjusted }\end{array}$ \\
\hline \multicolumn{3}{|l|}{ Wealth index } \\
\hline Lowest & $28.38(26.32,30.43)$ & $27.56(25.56,29.57)$ \\
\hline Low & $27.69(25.76,29.63)$ & $28.03(26.10,29.97)$ \\
\hline Middle & $23.45(21.45,25.46)$ & $23.70(21.69,25.70)$ \\
\hline High & $21.43(19.64,23.21)$ & $21.59(19.81,23.37)$ \\
\hline Highest & $15.78(14.15,17.41)$ & $15.79(14.17,17.41)$ \\
\hline
\end{tabular}

Table 5: Unadjusted and age-adjusted prevalence of Current Tobacco Smokers derived from logistic regression to current tobacco smoking (Sample size=9565)

Unadjusted and adjusted relationships of socio-demographic and economic variables to smoking status have been analyzed to identify the predictors of current tobacco smoking (Table 6). It has been found that for unadjusted case, rural respondents are significantly and more likely to smoke tobacco currently $(\mathrm{OR}=1.16,95 \% \mathrm{CI}=1.05,1.27)$. But when adjusted to all factors, rural respondents are found to be less likely to smoke but not significantly ( $\mathrm{OR}=0.90,95 \% \mathrm{CI}=0.79,1.04)$. Males are about 62.45 times more likely to smoke currently than females (unadjusted $\mathrm{OR}=62.45,95 \% \mathrm{CI}=49.42,78.91$ ) and after adjustment, they are 37.55 times more likely to smoke than females $(\mathrm{OR}=37.55,95 \% \mathrm{CI}=25.91,54.41)$. Respondents of youngest age group (15-24yrs) are less likely to smoke currently than all other age groups for both adjusted and unadjusted cases and also respondents with no formal schooling are most likely to smoke than respondents with all other level of education. Businessmen are 2.49 times more likely to smoke than employee (unadjusted $\mathrm{OR}=2.49,95 \% \mathrm{CI}=2.06,3.01$ ), farmers are 3.04 times more likely (unadjusted $\mathrm{OR}=3.04$, $95 \% \mathrm{CI}=2.50,3.71$ ), workers/day labours are 2.65 times more likely, and others are less likely to smoke currently than employee. Similar pattern has been found after adjusting with all factors. It has been also found that whether all factors are adjusted or not respondents with lowest wealth index are most likely to smoke than respondents of all other wealth index.

\begin{tabular}{|c|c|c|}
\hline \multirow[t]{2}{*}{ Socio-demographic factors } & \multicolumn{2}{|c|}{ Current Smokers } \\
\hline & $\begin{array}{c}\text { OR }(95 \% \text { CI }) \\
\text { Unadjusted }\end{array}$ & $\begin{array}{c}\text { OR }(95 \% \text { CI }) \\
\text { Adjusted }\end{array}$ \\
\hline \multicolumn{3}{|l|}{ Residence } \\
\hline Urban & 1 & 1 \\
\hline Rural & $1.162(1.06,1.28)$ & $0.90(0.79,1.04)$ \\
\hline \multicolumn{3}{|l|}{ Gender } \\
\hline Female & 1 & 1 \\
\hline Male & $61.66(48.80,77.92)$ & $37.55(25.91,54.41)$ \\
\hline \multicolumn{3}{|l|}{ Age } \\
\hline $15-24$ & 1 & 1 \\
\hline $25-34$ & $2.25(1.91,2.66)$ & $2.19(1.79,2.68)$ \\
\hline $35-44$ & $3.27(2.78,3.86)$ & $2.48(2.02,3.05)$ \\
\hline $45-54$ & $3.52(2.94,4.21)$ & $3.04(2.41,3.83)$ \\
\hline $55 \&$ above & $3.02(2.51,3.62)$ & $2.02(1.59,2.56)$ \\
\hline $\mathrm{p}$-value & $<0.001$ & $<0.001$ \\
\hline \multicolumn{3}{|l|}{ Educational Level } \\
\hline No formal schooling & 1 & 1 \\
\hline Less than primary school completed & $0.85(0.75,0.98)$ & $0.69(0.57,0.82)$ \\
\hline Primary school completed & $0.504(0.43,0.60)$ & $0.53(0.42,0.63)$ \\
\hline Less than secondary school completed & $0.52(0.45,0.59)$ & $0.52(0.42,0.63)$ \\
\hline Secondary school completed & $0.42(0.33,0.52)$ & $0.35(0.26,0.46)$ \\
\hline High school completed & $0.36(0.27,0.47)$ & $0.27(0.19,0.39)$ \\
\hline College/University completed \& higher & $0.52(0.41,0.67)$ & $0.26(0.19,0.36)$ \\
\hline p-value & $<0.001$ & $<0.001$ \\
\hline \multicolumn{3}{|l|}{ Occupation } \\
\hline Employment (government, non-government) & 1 & 1 \\
\hline Business ( small/large) & $2.47(2.05,2.99)$ & $1.29(1.04,1.59)$ \\
\hline Farming (land owner \& farmer) & $3.02(2.48,3.68)$ & $1.04(0.82,1.33)$ \\
\hline
\end{tabular}




\begin{tabular}{|l|c|c|}
\hline \multicolumn{1}{|c|}{ Socio-demographic factors } & \multicolumn{2}{c|}{ Current Smokers } \\
\cline { 2 - 3 } & $\begin{array}{c}\text { OR (95\% CI) } \\
\text { Unadjusted }\end{array}$ & $\begin{array}{c}\text { OR (95\% CI) } \\
\text { Adjusted }\end{array}$ \\
\hline $\begin{array}{l}\text { Agri or industrial worker/Daily labour/Other } \\
\text { self-employed }\end{array}$ & $2.65(2.23,3.16)$ & $1.23(0.98,1.54)$ \\
\hline Homemaker/ Housework & $0.04(0.03,0.05)$ & $0.40(0.25,0.65)$ \\
\hline Retired and unemployed (able/unable to work) & $0.81(0.62,1.06)$ & $0.67(0.48,0.92)$ \\
\hline Student/ Others & $0.54(0.43,0.68)$ & $0.84(0.64,1.11)$ \\
\hline p-value & $<0.001$ & $<0.001$ \\
\hline Wealth index & & 1 \\
\hline Lowest & $0.97(0.84,1.11)$ & $0.83(0.68,1.01)$ \\
\hline Low & $0.77(0.67,0.90)$ & $0.69(0.56,0.85)$ \\
\hline Middle & $0.69(0.60,0.80)$ & $0.61(0.49,0.75)$ \\
\hline High & $0.47(0.40,0.55)$ & $0.55(0.43,0.71)$ \\
\hline Highest & $<0.001$ & $<0.001$ \\
\hline p-value & & 1 \\
\hline
\end{tabular}

Note: $\mathrm{p}$-values are for trend in variables with more than two categories

Table 6: Unadjusted and adjusted odds ratios derived from logistic regression to current tobacco smoking (Sample size=9565)

\section{Discussion}

In this country representative cross-sectional study of Bangladesh, prevalence of current tobacco smoking is $23.19 \%$, of which $48.28 \%$ are male and $1.47 \%$ are female. Daily tobacco smoking is more prevalent in rural area (22.51\%) than urban area (19.85\%). Youngest subjects are less likely, whereas subjects with no formal education and those with lowest wealth-index are more likely to be current smokers than their counterpart. Businessmen, farmers and workers/day labours are more likely to be current smokers, and employee, students and respondents with other jobs are less likely to be current smokers.

The prevalence of tobacco smoking of this study reveals that about 37 million peoples with age 15 years and above are currently smoking in Bangladesh. However, the prevalence of smoking among males is found to be very high and higher than neibouring countries Pakistan [16] and Nepal [17]. It is a hope that due to social norms in Bangladesh tobacco smoking of females are not well accepted. The reported results also show the same scenario. But, this large proportion of males smoking tobacco may cause second hand smoking among females [18] as well as among children and non-smoker males.

In terms of regional differences, respondents from rural area are more likely to smoke tobacco. This might be for their ignorance about adverse effect of tobacco smoking, or they might not be aware about their own health like many other diseases [19,20]. Respondents belonging youngest age group have been found to smoke tobacco less. But the onset of earning age group is found to smoke more. The increase in prevalence among them might have indication about their job stress or family stress. Unless effective tobacco control measures are implemented soon, the future disease burden in Bangladesh will probably be influenced by the high level of smoking in young male adults.

Like other public health concerns, less educated people are more likely to smoke tobacco as they are less aware about health hazards and more likely to have higher degree of fatalism and overall risk taking behavior [21]. Same result has been found in India and Sri Lanka [22]. Government and nongovernment employee, retired persons, homemaker/ housework, retired and unemployed, and students/ others are less likely to smoke. On the other hand, businessmen, farmers, and day labours are more likely to smoke. Similar result has been reported for developed countries [23,24] and also other studies for Bangladesh [25]. Peoples with stressed job are more likely to find themselves in condition predisposing them to initiation of smoking tobacco. Similar pattern has been found with respect to wealth index. Respondents with lowest wealth index are most likely to smoke and with highest wealth index are least likely.

The major strengths of our study include large sample size, the coverage of men and women smokers, the coverage of both rural and urban areas, and the nationally representative population. However, there are several limitations that need to be addressed. The findings in this report are based on self- reports [12]. Furthermore, education categories were combined into broad groupings, which could have contributed to biased estimates in terms of the gradients observed. Nonetheless, these groupings provided greater precision than those used in earlier tobacco use research in Bangladesh [8-10]. The data used in constructing wealth index is based on limited number of asset variables, which might result in incomplete or under representing socioeconomic status. Some other variables like psychological variables could provide more predicting accuracy, but no such variable is available.

In conclusion, the results of this study reveal that tobacco smoking is strongly associated with social disadvantage, for example, low socio-economic status, less education. Giving it as public health priority, WHO Framework Convention on Tobacco Control should be implemented. In addition, a nationwide campaign is needed to educate people in rural area about the health risks of smoking tobacco. 


\section{Acknowledgement}

The authors would like to acknowledge the members of the GATS Collaborative Group.

\section{References}

1. Lim SS, Vos T, Flaxman AD, Danaei G, Shibuya K, et al. (2012) A comparative risk assessment of burden of disease and injury attributable to 67 risk factors and risk factor clusters in 21 regions, 1990-2010: a systematic analysis for the Global Burden of Disease Study 2010. Lancet 380: 2224-60.

2. Evaluation IfHMa. Global Burden of Disease (GBD) Visualizations.

3. Ng M, Freeman MK, Fleming TD, Robinson M, Dwyer-Lindgren L, et al. (2014) Smoking prevalence and cigarette consumption in 187 countries, $1980-2012$. JAMA 311: 183-92.

4. World Health Organization (2014) WHO Report on the global tobacco epidemic.

5. Alam DS, Jha P, Ramasundarahettige C, Streatfield PK, Niessen LW, et al. (2013) Smoking-attributablemortality in Bangladesh: proportional mortality study. Bull World Health Organ 91: 757-64.

6. Wu F, Chen Y, Parvez F, Segers S, Argos M, et al. (2013) A prospective study of tobacco smoking and mortality in Bangladesh. PLOS One 8: e58516.

7. Nargis N, Ruthbah UH, Hussain AK, Fong GT, Huq I, et al. (2014) The price sensitivity of cigarette consumption in Bangladesh: evidence from the International Tobacco Control (ITC) Bangladesh Wave 1 (2009) and Wave 2 (2010) Surveys. Tob Control 23 Suppl 1: i39-47.

8. Khan MM, Khan A, Kraemer A, Mori M (2009) Prevalence and correlates of smoking among urban adult men in Bangladesh: slum versus non-slum comparison. BMC Public Health 9: 149.

9. Kabir MA, Goh KL, Kamal SM, Khan MM (2013) Tobacco smoking and its association with illicit drug use among young men aged15-24 years living in urban slums of Bangladesh. PLoS One 9: e91618.

10. Kabir MA, Goh KL, Khan MM (2013) Adolescent Tobacco Use and Its Determinants: Evidence From Global Youth Tobacco Survey, Bangladesh 2007. Asia Pac J Public Health 27: NP1578-90.

11. Kabir MA, Goh KL, Khan MM (2013) Tobacco consumption and illegal drug use among Bangladeshi males: association and determinants. Am J Mens Health 7: 128-37.

12. Global Adult Tobacco Survey Collaborative Group (2010) Global Adult Tobacco Survey.

13. Giovino GA, Mirza SA, Samet JM, Gupta PC, Jarvis MJ, et al. (2012) Tobacco use in 3 billion individuals from 16 countries: an analysis of nationally representative cross-sectional household surveys. Lancet 380: 668-79.

14. Kalsbeek WD, Bowling JM, Hsia J, Mirza S, Palipudi KM, et al. (2010) The Global Adult Tobacco Survey: sample design and related methods.

15. Global Adult Tobacco Survey Bangladesh Report (2009) Global Adult Tobacco Survey.

16. Gilani SI, Leon DA (2013) Prevalence and sociodemographic determinants of tobacco use among adults in Pakistan: findings of a nationwide survey conducted in 2012. Population Health Matrics 11: 16.

17. Sreeramareddy CT, Ramakrishnareddy N, Kumar HNH, Sathian B, Arokiasamy JT (2011) Prevalence, distribution and correlates of tobacco smoking and chewing in Nepal: a secondary data analysis of Nepal Demographic and Health Survey-2006. Substance Abuse Treatment, Prevention, and Policy 6: 33.

18. Palupadi KM, Sinha DN, Choudhuri S, Mustafa Z, Andes L, et al. (2011) Exposure to tobacco smoke among adults in Bangladesh. Indian J Public Health 55: 210-9.

19. Rahman M, Islam A, M Islam (2008) Determinants of Knowledge and Awareness about AIDS: Urban -Rural Differentials in Bangladesh. The Int J Health 9: 2. 20. Uddin MJ, Choudhury MN (2008) Reproductive Health Awareness among Adolescent Girls in Rural Bangladesh. Asia Pac J Public Health 20: 117-28.

21. World Health Organization (2004) The Millennium Development Goals and Tobacco Control. Geneva, Switzerland.

22. Rani M, Bonu S, Jha P, Nguyen S, Jamjoum L (2003) Tobacco use in India: prevalence and predictors of smoking and chewing in a national cross sectional household survey. Tobacco Control 12 .

23. Gupta PC, Mehta HC (2000) Cohort study of all-cause mortality among tobacco users in Mumbai, India. Bull World Health Organ 78: 877-83.

24. Howard J (2004) Smoking is an occupational hazard. Am J Ind Med 46: 161-9.

25. Rahman MM, Karim MJ, Ahmad AJ, Suhaili AR, Ahmad SNW (2011) Prevalence and Determinants of smoking Behaviour among the Secondary School Teachers in Bangladesh. Int J Pub Health Res Special Issue: 25-32.

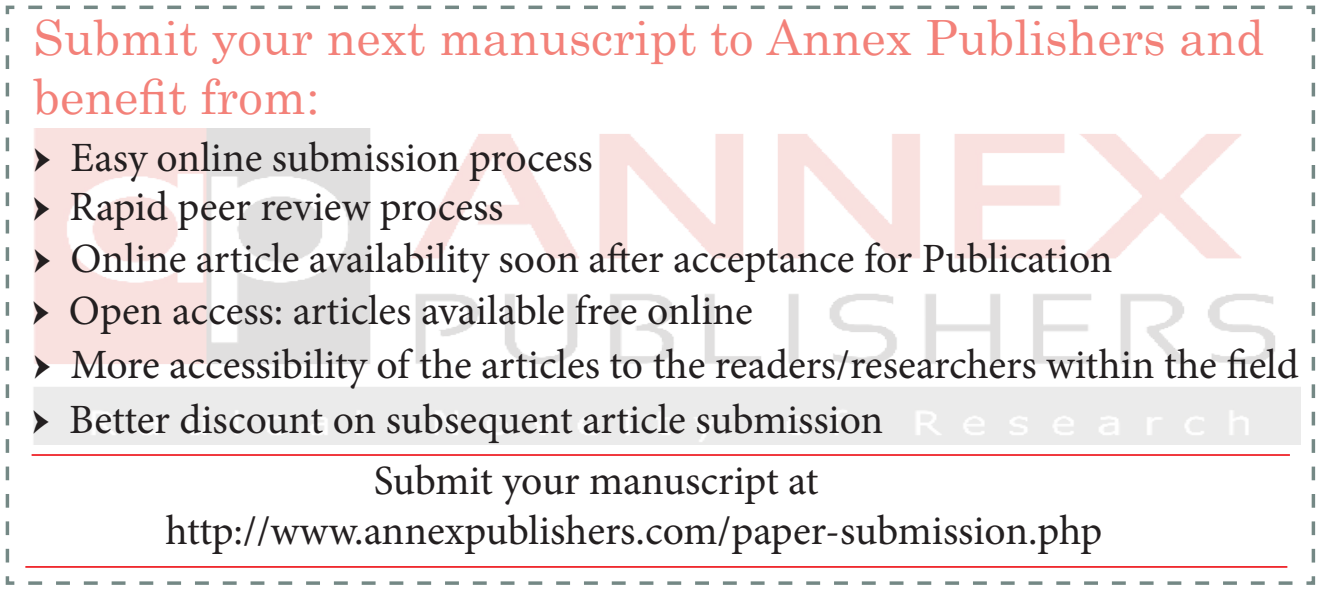

